\title{
COMPUTATIONAL ANALYSIS OF THE COLLAPSE BEHAVIOUR OF THIN-WALLED POLYGONAL STEEL BEAMS
}

\author{
Raffaele Ardito ${ }^{1}$ \\ ${ }^{1}$ Dept. of Civil and Environmental Engineering, Politecnico di Milano \\ Milan, Italy \\ e-mail: raffaele.ardito@polimi.it
}

Keywords: Local Buckling, Steel Structures, FE Analysis, Elastic-plastic Behavior, Thinwalled profiles, Imperfections

\begin{abstract}
The present paper is focused on the development of an accurate computational method, based on the Finite Element (FE) approximation, for predicting the collapse behavior of thin-walled polygonal steel beams subject to bending. The numerical model has been created using the software ABAQUS, and has been validated with experimental data obtained from the literature, concerning rectangular hollow section (RHS) in the four-point-bending situation. The model has been improved by means of a thorough study of material hardening, imperfections and residual stresses. The computational results of the analyses have been compared with the design procedures provided by Eurocode 3 (effective cross-section method and reduced stress method), in order to check their suitability.
\end{abstract}




\section{INTRODUCTION}

This paper describes the realization of a computational model for studying the flexural behaviour of polygonal, thin-walled sections at the ultimate limit state. This type of section is widely used as support of antennas in telecommunications, for lighting facilities and, in some cases, for small-scale wind turbines, see Figure 1.
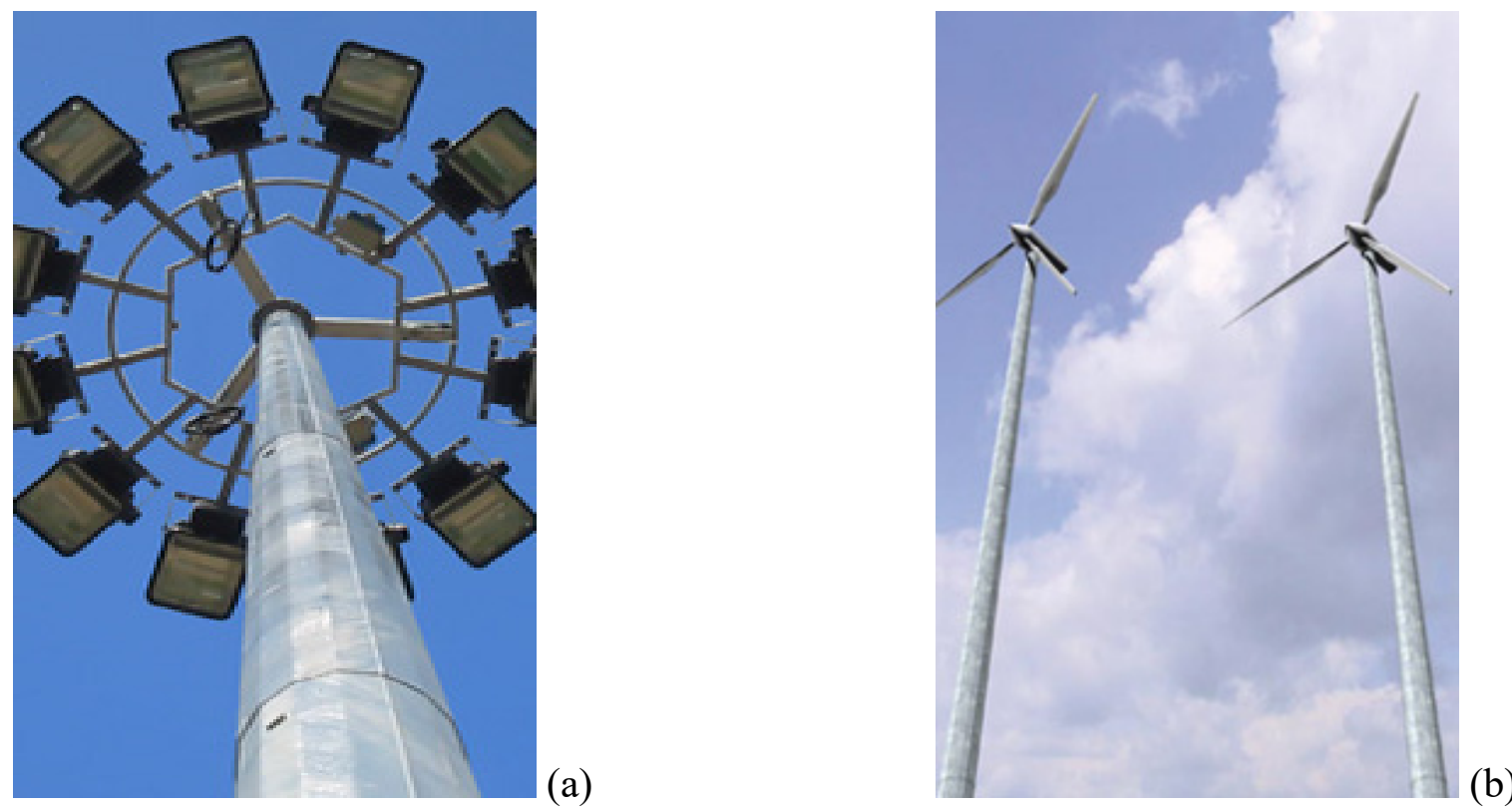

Figure 1: Examples of application of beams with thin-walled polygonal cross-section: (a) lighting tower; (b) mast for small-scale wind turbines.

The main goal is to evaluate the flexural strength of the examined sections. Because of the high slenderness, these structures are subjected to local buckling phenomenon, which complicates the determination of the load-carrying capacity. After a literature survey, we realized that many Authors have considered the load-carrying capacity of polygonal section, even though in most cases the reference problem was represented by axial load only (see e.g. [1], [2], [3]). The basic formulation for local buckling due to bending can be retrieved in the classical textbook [4], which reports the stability assessment for elastic plates rigidly jointed along their edges, in order to obtain open and closed thin-walled profiles. An attempt for the numerical simulation of local buckling can be found in [5], which reports also some results of an experimental campaign. The load carrying capacity of such structures is widely influenced by the presence of imperfections: some relevant information on residual stresses and geometric information can be retrieved in [6], [7] and [8], whereas the effect of metalworking process has been considered in [9].

In this paper, a computational model has been devised and the results of the analyses are compared to the expeditious procedures provided by Eurocode 3 (effective cross-section method and reduced stress method), checking their suitability. The numerical model has been created using the software ABAQUS [10], and has been validated with experimental data obtained from the literature, concerning rectangular hollow section (RHS) [11]. The model was developed, improving on it with the addition of material hardening, imperfections and residual stresses. The relevant data have been taken from Eurocode 3, literature or by experimental tests. The critical comparison with respect to experimental data has been used in order to assess the validity of the model in terms of load-curvature response. 
The analyses allowed us to understand the mechanisms by which the resistance develops in this type of sections. The comparison of the results of the numerical models with the Eurocode 3 ones has shown that often the legislation does not estimate accurately the resistance values. Moreover, in cases of tapered sections or lightened ones, the legislation proves to be deficient if not entirely inapplicable.

\section{PROBLEM FORMULATION}

The safety of slender steel beams can be endangered by the lack of stability in many different ways: global buckling refers to the overall collapse due to axial, bending, torsional or combined loads; distortional buckling is specially related to thin walled profiles, in terms of distortion of the cross-section with relative displacement of the nodes; local buckling, which is specifically treated in this paper, can be defined as the unstable behaviour of each single panel that constitute the cross-section, in the absence of global buckling and of relative displacement of the profile's nodes (which are basically fixed).

A simple treatment of local buckling is obtained by considering each panel as a plate subject to membrane loading and suitably constrained in order to account for the effect of adjacent plates. The axial compressive stress, which eventually lead to buckling, can be provided, for instance, by the overall bending moment, as shown in Figure 2.

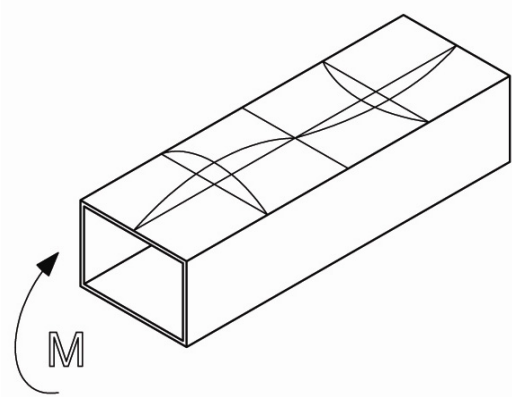

Figure 2: Local buckling of a thin-walled polygonal beam subject to bending moment $\mathrm{M}$.

The simplistic approach is represented by the computation of the Eulerian critical load for elastic behaviour, in the case of simply supported rectangular plate (see [4]). In that way, one finds that the critical stress can be computed as:

$$
\sigma_{E}=\frac{N_{E}}{t}=k_{\sigma} \frac{\pi^{2} E}{12\left(1-v^{2}\right)}\left(\frac{t}{b}\right)^{2}
$$

where: $b$ is the minimum width of the plate; $t$ is its thickness; $E$ and $v$ are the Young's modulus and the Poisson's ratio, respectively; $k_{\sigma}$ is the load factor, which depends on the elastic distribution of axial stress. For instance, in the case of uniform compression (as it happens in the upper flange in Figure 2), one finds $k_{\sigma}=4$; other values are reported in [4] for various non-uniform distributions.

A more reliable approach, which accounts for the post-critical behaviour, has been developed by Von Kármán on the basis of the concept of effective width [4]. Roughly speaking, the non-uniform stress distribution at plastic collapse is replaced by a piecewise constant distribution, with null value in the zone around the plate centre (which is assumed to be in the buckled configuration) whereas the lateral zones are subject to the yield stress for the given material. By enforcing the equivalence of the yield stress with the buckling tension of the effective zone, one finds: 


$$
\sigma_{c r}\left(b_{e f f}\right)=f_{y}=k_{\sigma} \frac{\pi^{2} E}{12\left(1-v^{2}\right)}\left(\frac{t}{b_{e f f}}\right)^{2}
$$

where $b_{\text {eff }}$ is the sum of the length of the lateral zones subject to the yield stress.

This approach does not take into account the effect of imperfections, which play a paramount role in the evaluation of critical load. Imperfections in steel structures may have different nature but they can be divided into a couple of categories: mechanical imperfections and geometrical imperfections. The former include the presence of residual stresses, due to the specific metalworking procedure (e.g. hot rolling or cold forming), and the non-uniform distribution of pristine yield limit, due e.g. to the folding process. An example of this kind of imperfection, which is quite important for polygonal sections, is reported in Figure 3.
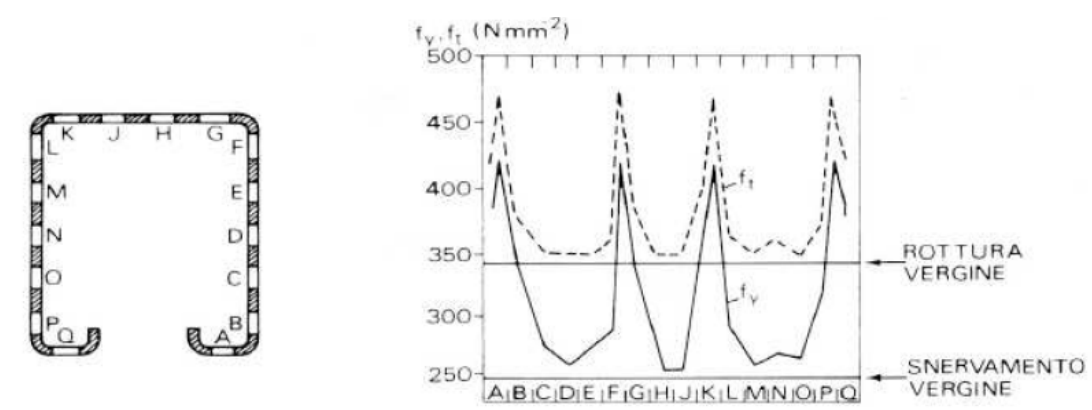

Figure 3: Typical distribution of the material properties on a cold-formed profile.

Geometric imperfections can be further divided into global defects (e.g. overall bow of the beam) and local defects (e.g. non-uniform thickness, lack of orthogonality, etc.). In the case of local buckling, the latter are by far more important. According to Eurocode 3 part 5, Annex C [12], the geometric imperfections can be modeled on the basis of the buckling modes (such as the one represented in Figure 2), with a recommended amplitude equal to $80 \%$ of the fabrication tolerances.

One theoretical approach, which accounts for the presence of imperfections, has been formulated by Winter [4]. The evaluation of the effective width is based on the dimensionless slenderness parameter $\lambda_{p}$ :

$$
\lambda_{p}=\sqrt{\frac{f_{y}}{\sigma_{E}}}
$$

where the critical stress is referred to the whole width, see Equation (1). Winter proposed the following expression, which is assumed in the current code provisions [12]:

$$
\frac{b_{e f f}}{b}=\left(1-\frac{0.22}{\lambda_{p}}\right) \frac{1}{\lambda_{p}} \leq 1
$$

A graphical comparison of the abovementioned approaches, in terms of the average collapse stress $\sigma_{L}$, is reported in Figure 4.

In the present paper, a computational approach is preferred in order to capture the collapse load for the whole cross-section. A 3D Finite Element (FE) model has been devised, as better specified in the next section, and a set of non-linear static analyses have been carried out, encompassing the non-linear geometric effects and the elastic-plastic behaviour. In any case, a preliminary step has been represented by linear buckling analyses, in order to evaluate the 
buckling modes which are used to model the geometric imperfections. The final loadcurvature response has been obtained by applying an incremental (step-by-step) analysis with arc-length control. Such a provision is necessary in order to account for strongly unstable behaviour, such as snap-back or snap-through phenomena, that are likely to occur in the present structural case, dominated by imperfections. The analyses have been repeated for different width-to thickness ratios, with the purpose of evaluating the collapse behaviour in various cases. It is worth noting that the width-to-thickness ratio represent the basic parameter used to decide whether the considered section is prone to local buckling.

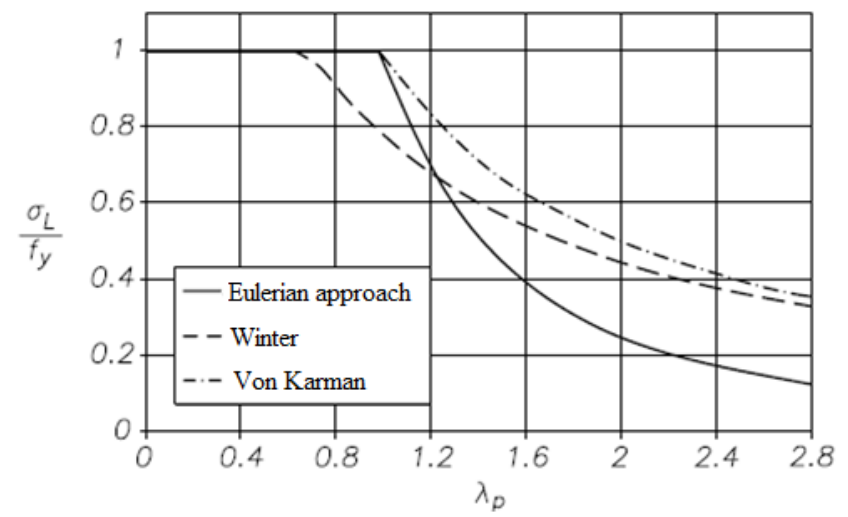

Figure 4: Stability curves for a simply supported plate (representative of a panel in thin-walled cross-sections) with reference to different theoretical approaches.

\section{RESULTS}

\subsection{Finite Element model}

The computational model has been tested with reference to experimental data, obtained at the University of Sidney, Australia, by Wilkinson and Hancock [11]. Those Authors have carried out a set of four-point bending tests on steel beams with rectangular hollow section (RHS). Several specimens with different thickness of the flanges have been tested, more specifically RHS 150x50x4, RHS 150x50x3 and RHS 150x50x2.3 (all the measurements are in millimeters); in that way, it was possible to evaluate the increasing sensitivity to imperfection for decreasing thickness. The scheme of the experiments is depicted in Figure 5a; conversely, Figure $5 \mathrm{~b}$ shows a simplified model of a beam subject to pure bending. In the following, both models will be considered, since the experiment model (Figure 5a) was used for a direct comparison with the tests and the simplified model (Figure 5b) could be adopted for further generalization and application to cross-sections of different shapes. The main outcome of the experimental campaign was a set of moment-curvature plots, which are normalized with respect to the elastic limit moment $\left(M_{p}\right.$, namely the moment that corresponds to the first attainment of yield stress in the worst point on the cross-section) and to the corresponding curvature $\left(\chi_{p}\right.$, given by the elastic limit moment $M_{p}$ divided by the elastic stiffness $\left.E I\right)$.

The Finite Element model was represented by a fine discretization of the RHS beams, using quadrilateral shell elements. In view of the very small thickness of the flanges, we chose a formulation which is suitable for thin shells, thus neglecting the shear deformation. Linear, 4node, elements have been used, with 5 degrees of freedom per node and reduced integration with hourglass mode control. The FE mesh has been chosen after the convergence analyses and is more refined in the central zone, as shown in Figure 6. The experiment model included also the discretization of the load posts and of the bearing devices. 

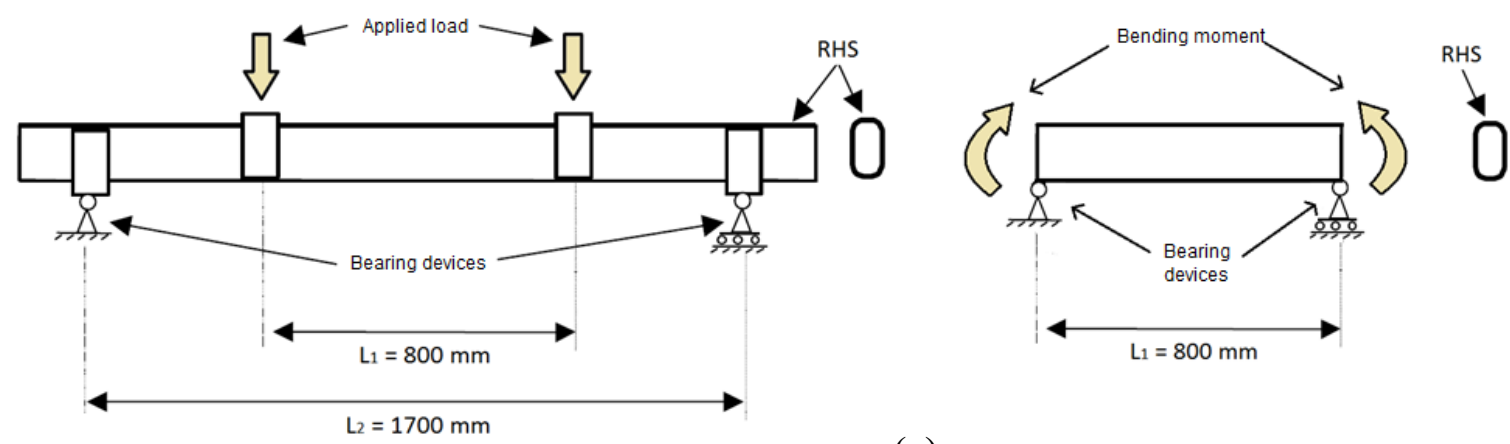

(a)

(b)

Figure 5: Scheme of the models considered in the computational analyses: (a) experiment model, which shares the geometric features with the tests in [11]; (b) simplified model, used for further generalization.

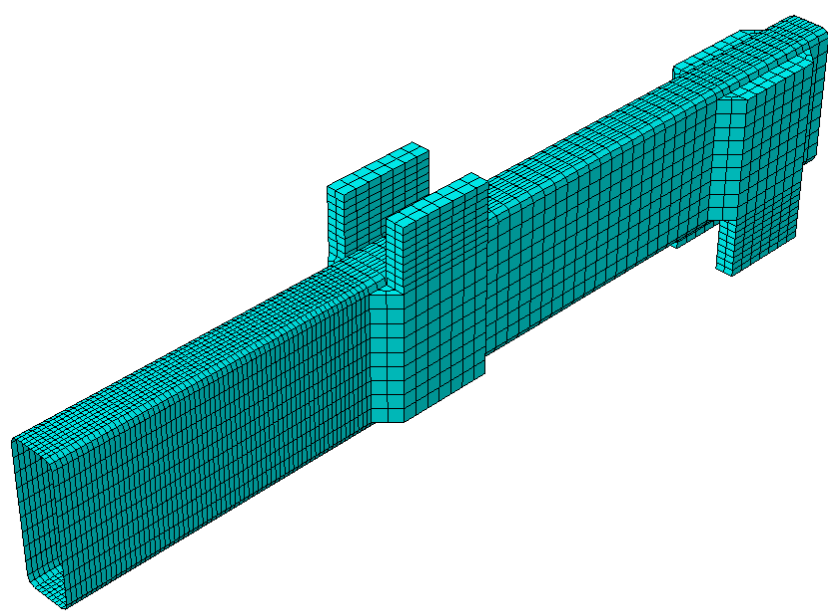

Figure 6: Typical FE model adopted in the non-linear analyses until collapse.

The preliminary step was represented by linear buckling analyses. In that way, it was possible to have an idea of the different buckling modes, shown in Figure 7: (a) local buckling due to compression on the upper flange; (b) local buckling due to bending on the vertical flanges; (c) local buckling due to shear on the lateral bays; (d) overall buckling. The knowledge of buckling modes was also necessary in order to include geometric imperfection in the subsequent non-linear analyses.

The collapse analyses, based on the arc-length method, included non-linearity from the geometric point of view and for material behavior. An elastic-plastic model, based on the Mises' yield function, has been adopted. Non-linear hardening was included, with the main purpose of simulating the actual behavior of steel specimens extracted from the tested structure [11]. It is worth noting that the material law was not uniform in space: indeed, due to fabrication process, the material close to the corners is more resistant and more brittle than the material in other locations. More specifically, three different zones have been considered: corners, horizontal flanges, vertical flanges (see Figure 8).

The optimal version of the FE model has been obtained after several parametric analyses. First, the effect of imperfections, both in terms of amplitude and in terms of shape, was considered. Nice results have been obtained by considering the imperfection as proportional to the first local buckling mode (see Figure 7a), with a typical amplitude equal to $1 / 250 \div 1 / 500$ of the beam's height. The effect of residual stresses was included as well: we finally adopted the typical values proposed by [6] and [7] (ranging between $0.08 f_{y}$ and $0.17 f_{y}$, in correspondence of the longitudinal weld and of other locations, respectively). 


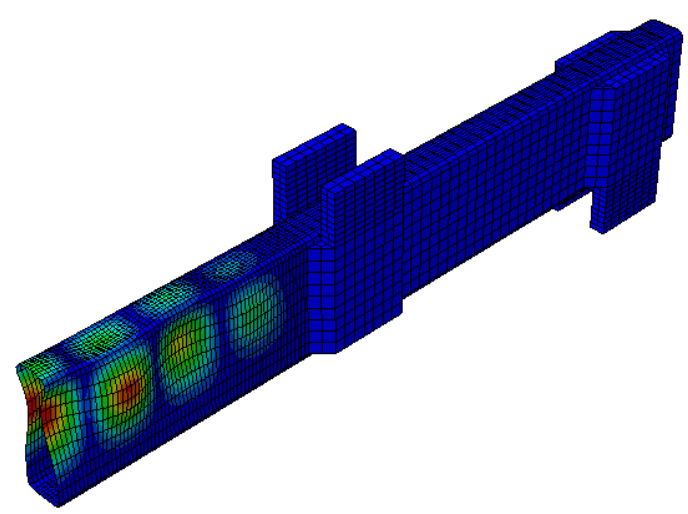

(a)
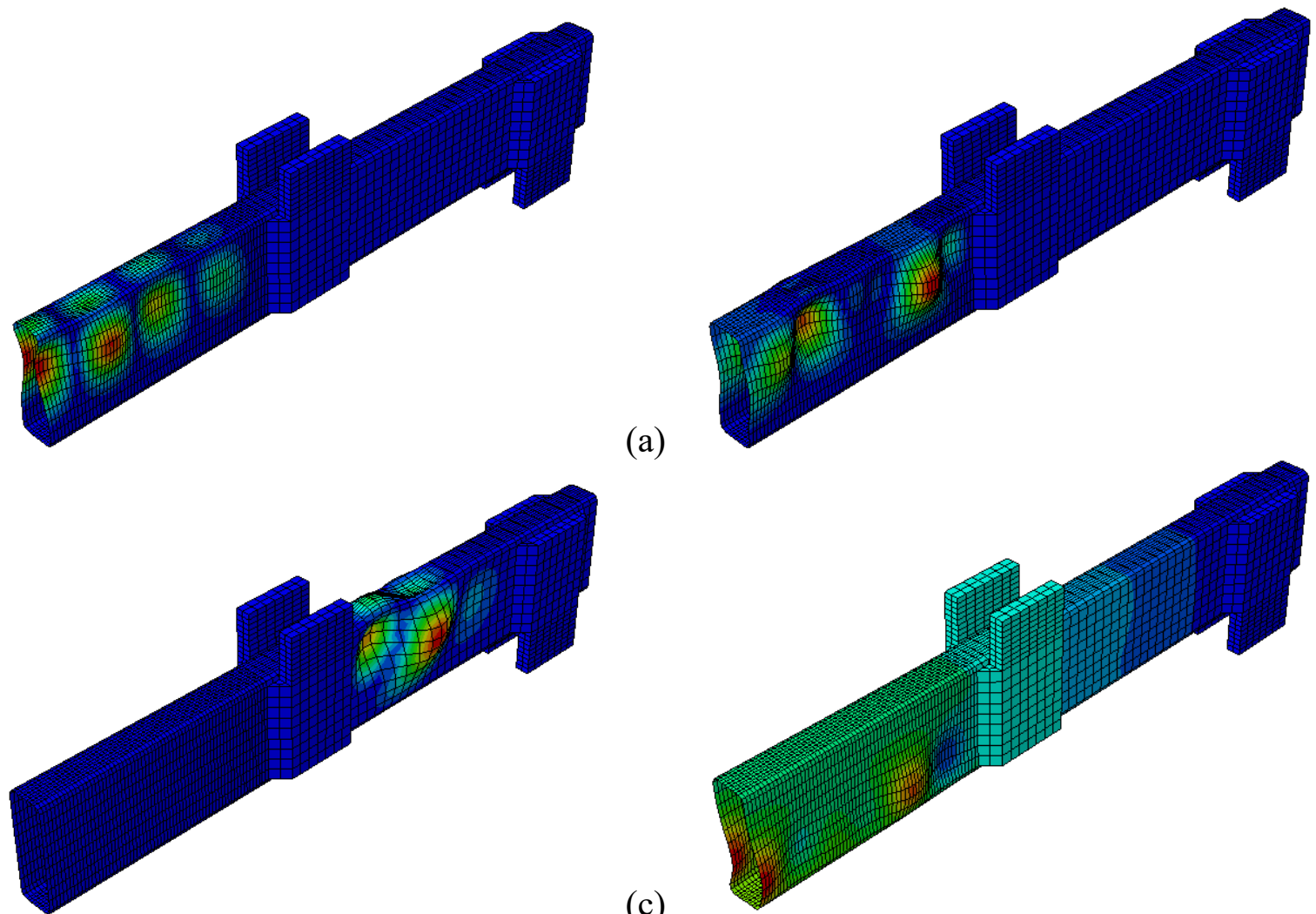

(b)

(c)

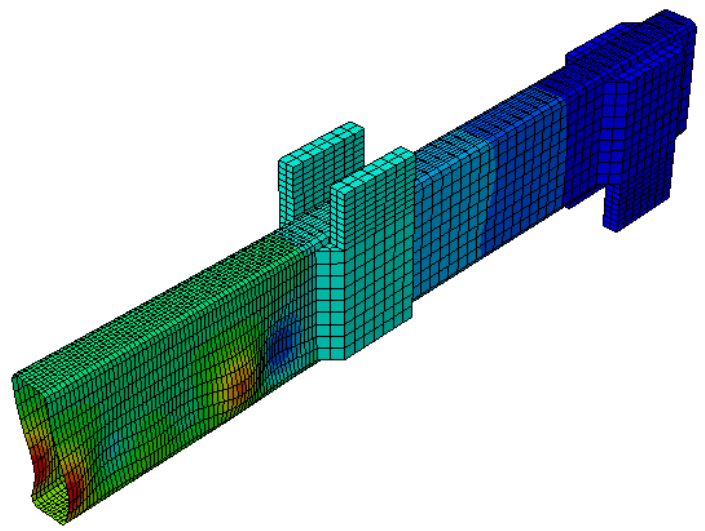

(d)

Figure 7: Results of the preliminary buckling analyses: (a), (b) local buckling for bending; (c) local buckling for shear; (d) overall buckling.

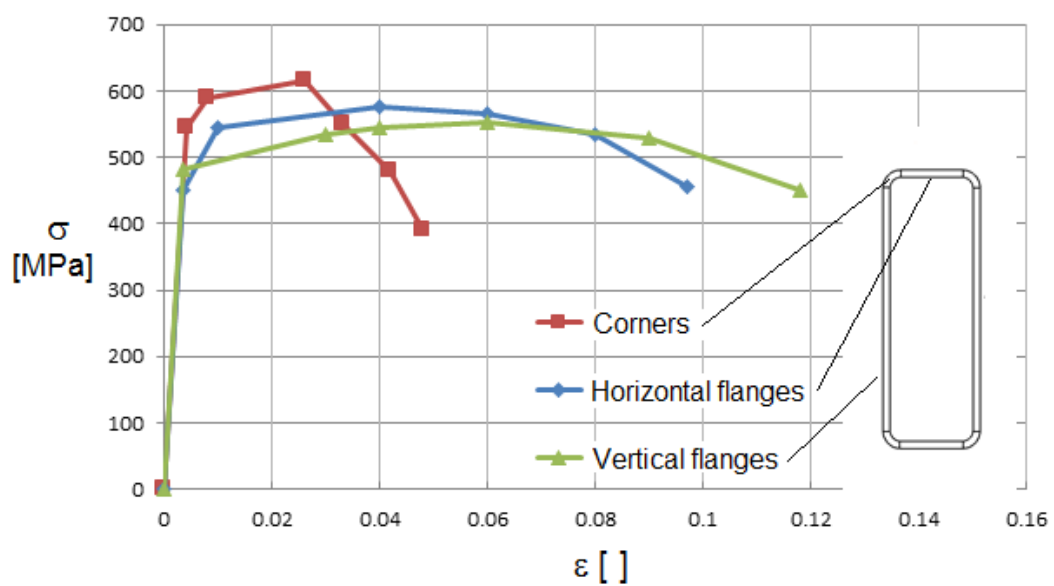

Figure 8: Uniaxial elastic-plastic material behavior for different zones of the RHS cross-sections.

\subsection{Comparison of load-curvature responses}

The optimal FE model, which did not include any fitting parameter, provided us the collapse load (see Figure 9 for the final deformed shape) and (after some post-processing) the load-curvature response (see Figure 10-12).

Generally speaking, satisfactory agreement between experimental and numerical data was obtained. The ultimate bending moment was captured with a reasonable relative error in all the considered cases; the ultimate curvature (as well as the initial stiffness) was very well simulated; also the deviation from linearity, owing to second-order geometric effects, was attained at the same load level with respect to the experimental outcomes. 


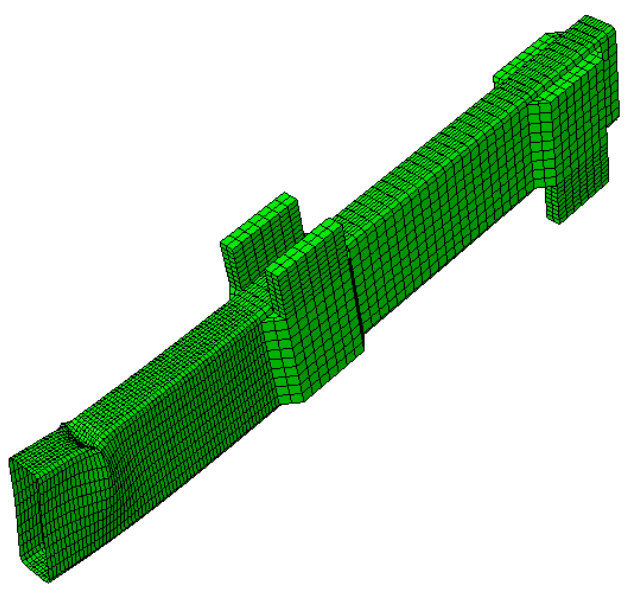

(a)

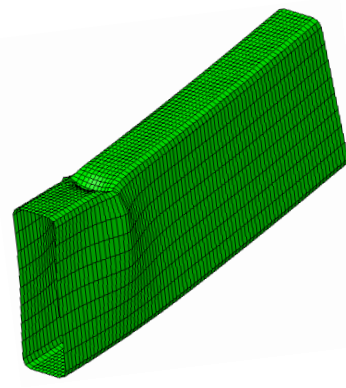

Figure 9: Deformed shape at collapse: (a) experiment model; (b) simplified model.

(b)

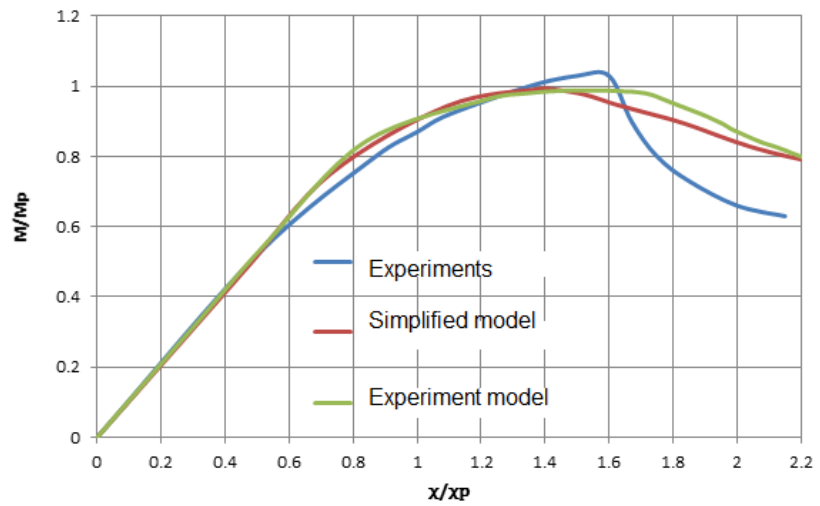

Figure 10: Load-curvature response for RHS 150x50x2.3.

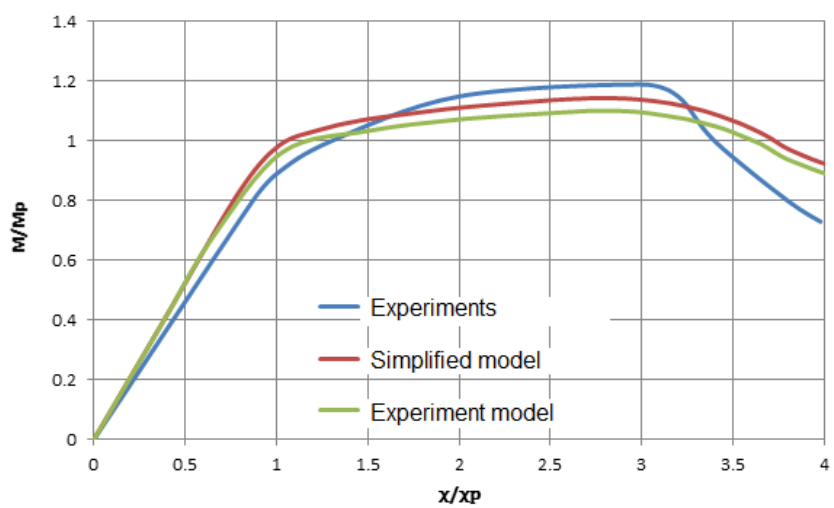

Figure 11: Load-curvature response for RHS 150x50x3.

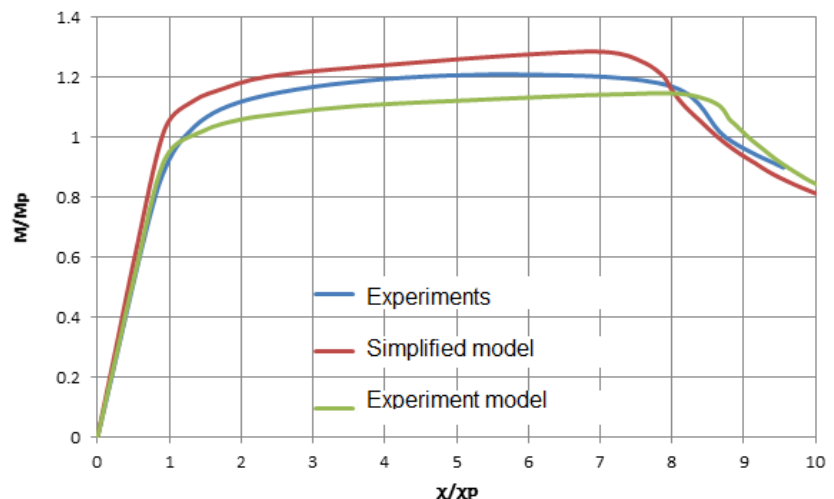

Figure 12: Load-curvature response for RHS 150x50x4. 
Some differences between the curves could be due to the spatial discontinuities of the constitutive law. In the real case, the stress-strain model is smoothly changing between the various parts of the section highlighted in Figure 8, conversely the FE model includes piecewise constant material laws. Another cause of discrepancies could be represented by the stochastic nature of imperfections, which turned out to play a paramount role in the definition of the collapse load.

All the analyses have been repeated by adopting the simplified model: as a matter of fact, some differences arose with respect to the experiment model. Even though the curves shared the same shape, the simplified model involved slightly larger ultimate loads. The difference could be explained by considering the effect of the loading posts, which have been completely modeled in the experiment model and are totally absent in the simplified model. However, both model revealed to be adequate to catch, with sufficient accuracy, the experimental response. This provided a sound validation of the computational models.

\section{CONCLUSIONS}

An optimized FE model for the collapse analyses of polygonal steel beams has been proposed and validated against reliable experimental data. Satisfactory agreement between the computational outcomes and the experimental data has been achieved, so that the computational model is basically ready to be used in other cases. As an example, the case of hexagonal or octagonal profiles could be considered, in order to execute the safety assessment of structures with practical interest.

The presence of a computational tool for that kind of structures is specifically important, since the application of simplified formulas, which can be retrieved in the Eurocode (EC) [15], is rather questionable for polygonal cross-section with six sides or more. As an example, we considered the theoretical computation of ultimate moment for three cases of RHS: the results are summarized in Table 1. One can easily realize that the formula proposed by the EC, though very simple to be used, finally yields poorly approximate results, which are not on the safe side (the ultimate moment is overestimated by the EC procedure). Some current research, which will be reported in a forthcoming paper, shows that the inaccuracy of EC formulas is even larger for sections with six sides or more

\begin{tabular}{|c|c|c|c|c|}
\hline Section & Class & $\begin{array}{l}M_{u}(E C) \\
{[k N \cdot m]}\end{array}$ & $\begin{array}{l}M_{u}(F E) \\
{[k N \cdot m]}\end{array}$ & $\begin{array}{c}\Delta \\
{[\%]}\end{array}$ \\
\hline RHS $150 \times 50 \times 2$ & 3 & 12.36 & 11.91 & +3.73 \\
\hline RHS $150 \times 50 \times 3$ & 1 & 23.05 & 20.51 & +12.38 \\
\hline RHS $150 \times 50 \times 4$ & 1 & 29.95 & 28.69 & +4.38 \\
\hline
\end{tabular}

Table 1: Comparison between the ultimate moment obtained via the numerical procedure (FE) and the formula proposed by the Eurocode (EC).

\section{REFERENCES}

[1] A. Godat, F. Legeron, D. Bazonga, "Stability investigation of local buckling behavior of tubular polygon columns under concentric compression," Thin-walled Struct., vol. 53, pp. 131-140, 2012.

[2] K.H. Law, L. Gardner, "Buckling of elliptical hollow section members under combined compression and uniaxial bending", J. of Constr. Steel Res., vol. 86, pp. 1-16, 
2013.

[3] A. Rossi, Z. Fawaz, K. Behdinam, "Numerical simulation of the axial collapse of thin walled polygonal section tubes", Thin-Walled Struct., vol. 43, pp. 1646-1661, 2005.

[4] T.V. Galambos, Guide to stability design criteria for metal structures $-5^{\text {th }}$ Edition", John Wiley \& Sons, New York, 1998.

[5] K.S. Wanniarachchi, M. Mahendran, "Design of rectangular hollow flange steel beams," Proc. of the 8th International Conference on Steel, Space and Composite Structures, Kuala Lumpur, Malaysia, pp. 393-400, 2006.

[6] B.W. Schafer, T. Pekoz, "Geometric imperfection and residual stresses for use in the analytical modeling of cold-formed steel members", Thirteen International Specialty Conference of Cold-Formed Steel Structures, Missouri U.S.A., 1996.

[7] B.W. Schafer, T. Pekoz, "Computational modeling of cold-formed steel: characterizing geometric imperfections and residual stresses", J. of Constr. Steel Res., vol. 47, pp. 193-210, 1998.

[8] R.B. Cruise, L. Gardner, "Residual stress analysis of structural stainless steel sections", J. of Constr. Steel Res., vol. 64, pp. 352-366, 2008.

[9] L. Gardner, N. Saari, F. Wang, "Comparative experimental study of hot rolled and cold formed rectangular hollow sections”. Thin-Walled Struct., vol. 48, pp. 495-507, 2010.

[10] Dassault Systémes Simulia, Abaqus 6.10 Analysis User's Manual. Providence, 2010.

[11] T. Wilkinson, G.J. Hancock, Finite element analysis of plastic rectangular hollow sections beams, Research report $N^{\circ}$ R792, Department of Civil Engineering, University of Sydney, Australia.

[12] EN 1993-1-5, Eurocode 3 - Design of Steel Structures. Part 1-5: Plated Structural Elements, 2007. 